\title{
Survival in a Chilean Cohort of Thymic Tumors
}

\author{
Mauricio Salinas $^{1^{*}}$, Mozart Liessi ${ }^{1}$, Patricio Vallejo ${ }^{2}$, Cristian Ibarra ${ }^{2}$ \\ ${ }^{1}$ School of Medicine, Universidad de Chile, Santiago, Chile \\ ${ }^{2}$ Instituto Nacional del Tórax, Santiago, Chile \\ Email: *Mrsf24@hotmail.com
}

How to cite this paper: Salinas, M., Liessi, M., Vallejo, P. and Ibarra, C. (2019) Survival in a Chilean Cohort of Thymic Tumors. Journal of Cancer Therapy, 10, 510-517. https://doi.org/10.4236/jct.2019.107042

Received: January 22, 2019

Accepted: July 7, 2019

Published: July 10, 2019

Copyright $\odot 2019$ by author(s) and Scientific Research Publishing Inc. This work is licensed under the Creative Commons Attribution International License (CC BY 4.0).

http://creativecommons.org/licenses/by/4.0/ (c) (i) Open Access

\begin{abstract}
Introduction: Thymus neoplasm is rare tumors and it has low mortality. There are scarce publications in Latin-American about this topic. We present a Chilean casuistic. Method: Retrospective cohort of every patient's thymic epithelial tumors diagnosed in Instituto Nacional del Tórax, Santiago, Chile, between January 2007 and June 2015. Information was obtained from pathological and clinical registries. Death date was achieved from national registry. We report epidemiological data, clinical manifestations, histology type, Masaoka and WHO classification, therapies and survivor. Results: Sixty-five patients were included, with mean age 58 years and 36.9\% men. Myasthenia gravis was present in $24(36.9 \%)$ of patients. Ninety four percent of patients had a surgical biopsy. There were 60 thymomas and 5 thymic carcinomas. Most of tumors were stage I and II in Masaoka classification and type B in WHO classification. There were no deaths at 30 days. Nosocomial pneumonia was the most common complication. Conclusions: In this thymic tumor cohort there are more women and they are older than previously reported. Myasthenia gravis prevalence was similar. The survival of patients with thymomas was excellent.
\end{abstract}

\section{Keywords}

Thymoma, Thymus Neoplasms, Thymus Gland

\section{Introduction}

The epithelial neoplasms of the thymus correspond to primary tumors of the mediastinum, derivate from the thymic epithelium. They are rare tumors, which present in different series, annual incidence rates from 0.9 to 2.3 per million inhabitants [1]. Thymoma corresponds to the primary mediastinal tumor most commonly presented [2].

The natural history of the disease may include asymptomatic patients discov- 
ered incidentally or presented as aggressive malignant tumors in terms of growth and dissemination [3]. The prognosis is good in comparison to other types of cancer, with survival rates of $84 \%$ at one year and $64 \%$ at five years [1].

In patients with thymus tumors, the coexistence of other diseases should be considered, especially myasthenia gravis, found in approximately $40 \%$ of the thymomas. There has been described other diseases such as: pure aplasia of red blood cells, parathyroid adenoma and hypogammaglobulinemia [4].

There are scarce publications in Latin-American, in the last 20 years. In this article we present the different characteristics and survivals of a Chilean cohort of patients with thymic tumors.

\section{Methods}

We did a retrospective study, which was developed at the Instituto Nacional del Tórax (INT) in Santiago, Chile. The list of patients was obtained from the pathological registry, with all the biopsies diagnosis of thymoma or thymic carcinoma, from 2007 to 2015.

\subsection{Histopathology}

The histological samples were analyzed by certified pathologists from the Instituto Nacional del Tórax. The neoplasms of the thymus were classified as a thymoma or thymocarcinoma. Other types of thymus neoplasms were excluded from this analysis.

The selected thymomas were classified according to recommendation of the World Health Organization (WHO), as A, AB or B [5]. In the surgically removed tumors, it was stated if there was capsule penetration, lymph nodes compromise or if there was an invasion to the adjacent organs. Immunohistochemically studies were performed according to the pathologist's criteria.

\subsection{Clinical Information}

The register of the patients was obtained from physical and electronic. Demographic and clinical information was registered, with emphasis in: the presence and treatment of myasthenia gravis, the surgical technique performed, the post-surgery complications and the result from the surgery in relation to the tumor resection. The classification of the thymus neoplasms was done according to the modified Masaoka classification [6].

\subsection{Adjuvant Therapy}

In our center, all patients were presented to the oncological committee to decide chemotherapy or radiotherapy indication.

In the time frame analyzed, patients received chemotherapy with platinum plus etoposide chemotherapy and/or conventional radiotherapy. All this information was recorded and registered as well as the intention of the adjuvant therapy, which could've been curative or palliative. 


\subsection{Statistical Analysis}

The description of the numerical variables was made using averages and standard deviation with continuous variables. Absolute and relative frequencies were used with categorical variables.

The date of death was obtained from the national Chilean registry. The cohort was followed until January 1, 2017.

The survival rate is presented using the Kaplan Meier graphs. To comparison between the groups Log-rank test was performed. For multiple comparisons, bonferroni correction was utilized. A level of signification of 0.05 with to 2 tails was considered.

All analysis was done in Stata 14.0.

\section{Results}

Information was obtained from the results of 65 patients, between the years 2007 and 2015. More than 90 percent of the patients had a surgical biopsy.

The patients had age average of 58 years old; $37 \%$ male subjects. About the presentation symptoms, $36.9 \%$ of patients showed myasthenia gravis. There were no cases of Sjogren, red blood cells aplasia or hypogammaglobulinemia.

Sixty patients had thymoma-tumors and only five patients corresponded to thymic carcinomas. Characteristics of the patients were summarized in Table 1.

About the Masaoka classification, more than half of the tumors corresponded to stages I and II. According to WHO classification, most of cases were type B. One patient had all thymoma types areas in the biopsy, therefore, it was classified as mixed type (Table 2).

The most common complication seen was nosocomial pneumonia in $9.8 \%$ of the patients. There was no death related to the surgery.

Table 1. Baseline characteristics.

\begin{tabular}{cc}
\hline Age & Value $^{*}$ \\
Men & $57.9 \pm 12.9$ years \\
Symptoms & $24(36.9 \%)$ \\
Cough & \\
Dyspnea & $7(10.8 \%)$ \\
Pain & $14(21.5 \%)$ \\
Cava superior syndrome & $10(15.4 \%)$ \\
Myasthenia gravis & $1(4.3 \%)$ \\
Asymptomatic & $24(36.9 \%)$ \\
Surgical biopsy & $19(29.2 \%)$ \\
Thymoma & $61(93.8 \%)$ \\
\hline
\end{tabular}

${ }^{\star}$ Mean (SD) for continuous variables; $\mathrm{n}(\%)$ for categorical variables. 
Table 2. Thymic tumors stages.

\begin{tabular}{cc}
\hline & $\mathrm{n}(\%)$ \\
\hline Masaoka & $23(35.4 \%)$ \\
Stage I & $18(27.7 \%)$ \\
Stage II & $11(16.9 \%)$ \\
Stage III & $13(20.0 \%)$ \\
Stage IV & \\
WHO (n $=60)$ & $4(6.7 \%)$ \\
A & $9(15.0 \%)$ \\
AB & $46(76.7 \%)$ \\
B & $1(1.6 \%)$ \\
Mixed &
\end{tabular}

Three (12.5\%) of the twenty-four patients with myasthenia, presented myasthenic crisis during the post-operatory period; myasthenic crisis were treated using steroids and plasmapheresis with complete recovery in all patients.

In $49(80.3 \%)$ patients a total resection of the tumor was accomplished according to the anatomopathological review of the biopsy.

Nineteen patients were treated via complementary radiotherapy. In 12 (63.1\%) patients the radiotherapy was done with curative intention. Twelve patients (17.2\%) received chemotherapy, only one of them with curative intention (Table 3).

Table 3. Treatment and complications.

$\mathrm{n}(\%)$

\begin{tabular}{cc}
\hline Surgery outcome $(\mathrm{n}=60)$ & $49(80.3 \%)$ \\
Complete resection (R0) & $1(1.6 \%)$ \\
Microscopic positive margin (R1) & $11(18.1 \%)$ \\
Macroscopic positive margin (R2) & $3(12.5 \%)$ \\
Post-operative myasthenic crisis ${ }^{*}$ & $0(0 \%)$ \\
Mortality (30 days) & \\
Perioperative complications & $6(9.8 \%)$ \\
Nosocomial pneumonia & $1(1.6 \%)$ \\
Mediastinitis & $2(3.3 \%)$ \\
Wound infection & $1(1.6 \%)$ \\
Chylothorax & $1(1.6 \%)$ \\
Hemothorax & $12(18.4 \%)$ \\
Chemotherapy & $1(8.3 \%)$ \\
Curative intention & $11(91.7 \%)$ \\
Palliative intention & $19(29.2 \%)$ \\
Radiotherapy & $12(63.1 \%)$ \\
Curative intention & $7(36.8 \%)$ \\
Palliative intention &
\end{tabular}

*over 24 patients with myasthenia. 
Averages survival were 12 months for the thymic carcinoma group and 164 months in the thymoma group (Figure 1).

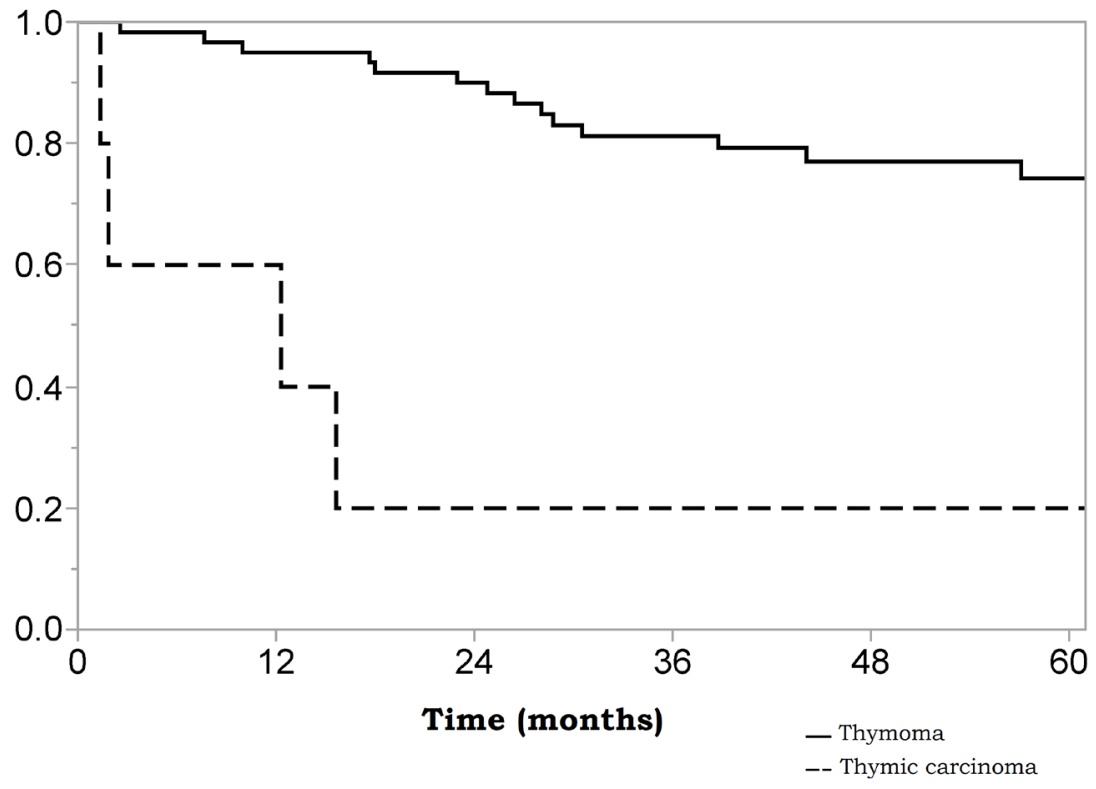

Figure 1. Survival of patients with thymoma and thymic carcinoma.

Averages survival for Masaoka stages 1, 2, 3 and 4 were 144, 72, 53 and 23 months respectively (Figure 2), with statistical significance between stages 1 and 3 ( $\mathrm{p}<0.01)$ and stages 1 and $4(\mathrm{p}<0.01)$. There were no survival differences by WHO classification.

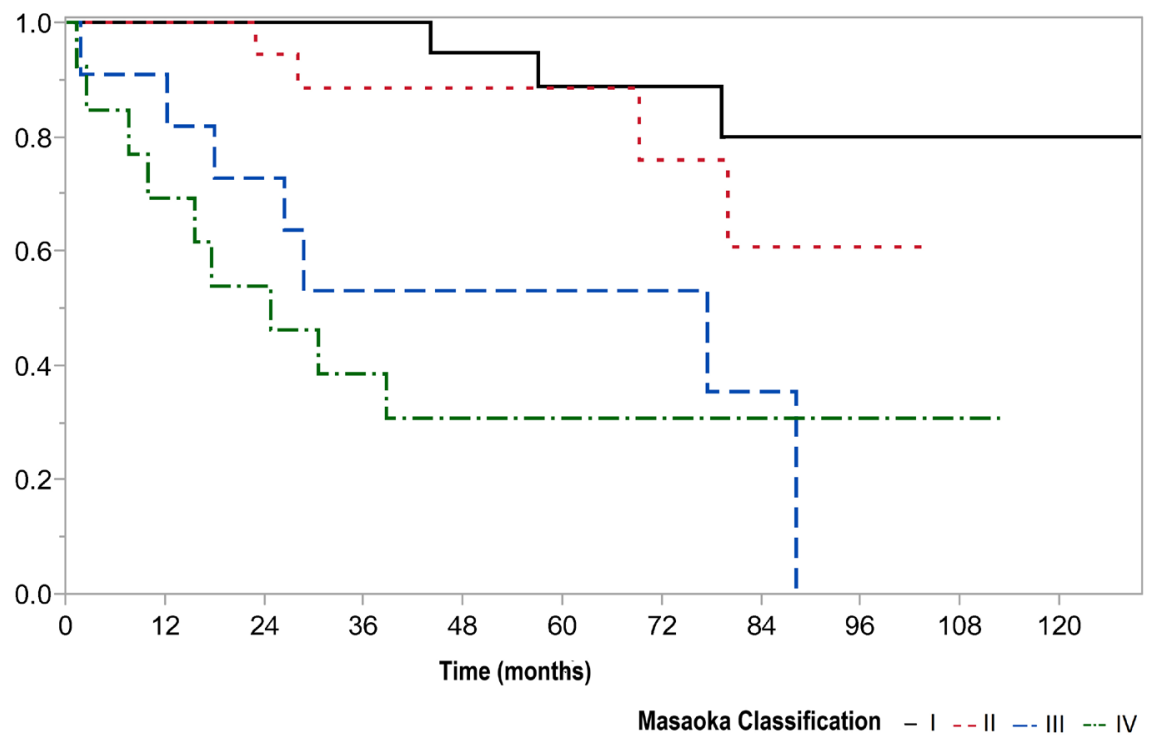

Figure 2. Survival of patients according to Masaoka classification.

\section{Discussion}

In this article we presented a cohort of a relatively big number of patients diag- 
nosed with thymic neoplasms, which represent a group of unusual malignant neoplasms [7]. The true incidence rate of the thymomas is yet unknown but represents approximately $47 \%$ of the tumors from the anterior mediastinum [8].

Thymomas may be asymptomatic and discovered by accident. Depending on the series of patients, up to $40 \%$ of the patients had local symptoms, which may include chest pains, cough and dyspnea by compression of the airway or by neuromuscular effect from the myasthenia gravis [9]. The paraneoplastic myasthenia gravis has been described in $30 \%-40 \%$ of the cases of thymoma [10]. In this series, almost one third of the total were asymptomatic, and another third were studied within the context of the myasthenia. There were no cases with paraneoplastic syndrome.

Thymomas might be classified by histological subtypes such as $\mathrm{A}, \mathrm{B}$ and $\mathrm{AB}$, but in the last group, $30 \%$ present histological heterogeneity [11]. This constitutes a problem for the pathologist's classification. In the series presented, the majority was classified as part of the B group, but one case had different areas in the tumor which presented the three subtypes and could not be classified according to the WHO recommendation.

Epithelial tumors of the thymus are distinguished by the invasive local growth and pleural dissemination, while the lymphatic and distant metastasis is very rare in the presence of a thymoma $(<5 \%)$. For thymic carcinoma, metastasis may reach more than $10 \%$ of patients and can explain a much worse prognosis [12] [13] [14].

In the series showed, survival rate of the stage IV thymomas and thymic carcinomas was poor compared to the thymomas in early stages. The histopathology of thymomas has also been described as a prognosis factor [5].

In an advanced thymoma or thymic carcinoma, patients may require multimodal therapies, which should include either induction or adjuvant chemotherapy, extended resections and post-operatory radiotherapy [15] [16] [17]. In the period recorded, the recommended drugs for this kind of neoplasms were not available at our center. Despite this, the survivals reported are concordant with what has been shown in the international literature.

The study has multiple limitations, including retrospective analysis, lack of standardized follow up of patients and chemotherapy used. The study was done in only one center, which receives most of the patients in the country with this kind of tumor, although the total number of thymic tumors is not known.

\section{Conflicts of Interest}

The authors do not declare conflicts of interest.

\section{References}

[1] Siesling, S., van der Zwan, J.M., Izarzugaza, I., Jaal, J., Treasure, T., Foschi, R., et al. (2012) Rare Thoracic Cancers: Including Peritoneum Mesothelioma. European Journal of Cancer, 48, 949-960. https://doi.org/10.1016/j.ejca.2012.02.047

[2] Qu, Y.-J., Liu, G.-B., Shi, H.-S., Liao, M.-Y., Yang, G.-F. and Tian, Z.-X. (2013) 
Preoperative CT Findings of Thymoma Are Correlated with Postoperative Masaoka Clinical Stage. Academic Radiology, 20, 66-72. https://doi.org/10.1016/j.acra.2012.08.002

[3] Scorsetti, M., Leo, F., Trama, A., D’Angelillo, R., Serpico, D., Macerelli, M., et al. (2016) Thymoma and Thymic Carcinomas. Critical Reviews in Oncology/Hematology, 99, 332-350. https://doi.org/10.1016/j.critrevonc.2016.01.012

[4] Rosenow, E.C. and Hurley, B.T. (1984) Disorders of the Thymus. A Review. Archives of Internal Medicine, 144, 763-770. https://doi.org/10.1001/archinte.1984.00350160117020

[5] Marx, A., Strobel, P., Bavde, S.S., Chalabreysse, L., Chan, J.K., Chen, G., et al. (2014) ITMIG Consensus Statement on the Use of the WHO Histological Classification of Thymoma and Thymic Carcinoma: Refined Definitions, Histological Criteria, and Reporting. Journal of Thoracic Oncology, 9, 596-611.

https://doi.org/10.1097/JTO.0000000000000154

[6] Masaoka, A. (2010) Staging System of Thymoma. Journal of Thoracic Oncology, 10, S304-S312. https://doi.org/10.1097/JTO.0b013e3181f20c05

[7] Detterbeck, F. and Parsons, A. (2008) Thymic Tumors: A Review of Current Diagnosis, Classification, and Treatment. In: Patterson, G.A., Deslauriers, J., Lerut, A., Luketich, J.D., Rice, T.W. and Pearson, F.G., Eds., Thoracic and Esophageal Surgery, 3rd Edition, Elsevier, Philadelphia, PA, 1589-1614. https://doi.org/10.1016/B978-0-443-06861-4.50134-9

[8] Chen, J., Weisbrod, G.L. and Herman, S.J. (1988) Computed Tomography and Pathologic Correlations of Thymic Lesions. Journal of Thoracic Imaging, 3, 61-65. https://doi.org/10.1097/00005382-198801000-00010

[9] Detterbeck, F.C. and Parsons, A.M. (2011) Management of Stage I and II Thymoma. Thoracic Surgery Clinics, 21, 59-67. https://doi.org/10.1016/j.thorsurg.2010.08.001

[10] Lancaster, E. (2014) Paraneoplastic Disorders in Thymoma Patients. Journal of Thoracic Oncology, 9, S143-S147. https://doi.org/10.1097/JTO.0000000000000300

[11] Moran, C.A. and Suster, S. (2000) On the Histologic Heterogeneity of Thymic Epithelial neoplasms. Impact of Sampling in Subtyping and Classification of Thymomas. American Journal of Clinical Pathology, 114, 760-766. https://doi.org/10.1309/CYJH-9RXM-P2PK-120J

[12] Thomas, C.R., Wright, C.D. and Loehrer, P.J. (1999) Thymoma: State of the Art. Journal of Clinical Oncology, 17, 2280-2289. https://doi.org/10.1200/JCO.1999.17.7.2280

[13] Koga, K., Matsuno, Y., Noguchi, M., Mukai, K., Asamura, H., Goya, T., et al. (1994) A Review of 79 Thymomas: Modification of Staging System and Reappraisal of Conventional Division into Invasive and Non-Invasive Thymoma. Pathology International, 44, 359-367. https://doi.org/10.1111/j.1440-1827.1994.tb02936.x

[14] Hosaka, Y., Tsuchida, M., Toyabe, S., Umezu, H., Eimoto, T. and Hayashi, J. (2010) Masaoka Stage and Histologic Grade Predict Prognosis in Patients with Thymic Carcinoma. The Annals of Thoracic Surgery, 89, 912-917. https://doi.org/10.1016/j.athoracsur.2009.11.057

[15] Kondo, K. and Monden, Y. (2003) Therapy for Thymic Epithelial Tumors: A Clinical Study of 1,320 Patients from Japan. The Annals of Thoracic Surgery, 76, 878-884. https://doi.org/10.1016/S0003-4975(03)00555-1

[16] Lucchi, M., Melfi, F., Dini, P., Basolo, F., Viti, A., Givigliano, F., et al. (2006) 
Neoadjuvant Chemotherapy for Stage III and IVA Thymomas: A Single-Institution Experience with a Long Follow-Up. Journal of Thoracic Oncology, 1, 308-313. https://doi.org/10.1016/S1556-0864(15)31586-0

[17] Wright, C.D. (2010) Extended Resections for Thymic Malignancies. Journal of Thoracic Oncology, 5, S344-S347. https://doi.org/10.1097/JTO.0b013e3181f20eb3 